\title{
Rapid Distinction and Semiquantitative Analysis of THC and CBD by Silver-Impregnated Paper Spray Mass Spectrometry
}

Si Huang, Frank W. Claassen, Teris A. van Beek, Bo Chen, Jianguo Zeng, Han Zuilhof,* and Gert IJ. Salentijn*

Cite This: Anal. Chem. 2021, 93, 3794-3802

Read Online

ABSTRACT: The control over the amount of psychoactive THC ( $\Delta$-9tetrahydrocannabinol) in commercial cannabidiol (CBD) products has to be strict. A fast and simple semiquantitative $\mathrm{Ag}(\mathrm{I})$-impregnated paper spray mass spectrometric method for differentiating between THC and CBD, which show no difference in standard single-stage or tandem MS, was established. Because of a different binding affinity to $\mathrm{Ag}(\mathrm{I})$ ions, quasi-molecular $\mathrm{Ag}(\mathrm{I})$ adducts $[\mathrm{THC}+\mathrm{Ag}]^{+}$and $[\mathrm{CBD}+\mathrm{Ag}]^{+}$at $\mathrm{m} / z 421$ and 423 give different fragmentation patterns. The product ions at $\mathrm{m} / z 313$ for THC and $\mathrm{m} / z 353$ and 355 for $\mathrm{CBD}$ can be used to distinguish THC and CBD and to determine their ratio. Quantification of THC/CBD ratios in commercial CBD oils was
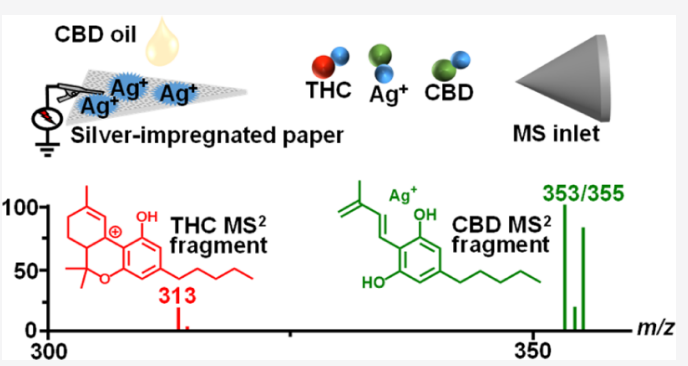
accomplished with a low matrix effect $(-2.2 \pm 0.4 \%$ for THC and $-2.0 \pm 0.3 \%$ for CBD $)$. After simple methanol extraction (recovery of $87.3 \pm 1.2 \%$ for THC and $92.3 \pm 1.4 \%$ for CBD), $\mathrm{Ag}(\mathrm{I})$-impregnated paper spray analysis was employed to determine this ratio. A single run can be completed in a few minutes. This method was benchmarked against the UHPLC-UV method. Ag(I)impregnated paper spray MS had the same working range $(\mathrm{THC} / \mathrm{CBD}=0.001-1)$ as UHPLC-UV analysis $\left(R^{2}=0.9896\right.$ and $R^{2}=$ 0.9998, respectively), as well as comparable accuracy ( -2.7 to $14 \%$ ) and precision (RSD $1.7-11 \%$ ). The method was further validated by the analysis of 10 commercial oils by $\mathrm{Ag}(\mathrm{I})$-impregnated paper spray MS and UHPLC-UV analysis. Based on the determined relative concentration ratios of THC/CBD and the declared CBD concentration, 6 out of 10 CBD oils appear to contain more THC than the Dutch legal limit of $0.05 \%$.

$\mathrm{C}$ annabis (Cannabis sativa L.) has been cultivated for medicinal, recreational, and industrial purposes since ancient times and remains a widely cultivated plant. ${ }^{1}$ Among its identified cannabinoids, $\Delta$ 9-tetrahydrocannabinol (THC) and cannabidiol (CBD), and their carboxylated forms, $\Delta 9$ tetrahydrocannabinolic acid (THCA) and cannabidiolic acid (CBDA), are the most sought after by people because of their psychoactive and therapeutic effects. ${ }^{2}$ THC is responsible for the psychoactive effects and has some potential analgesic, antiemetic, antikinetosic, and appetite-stimulating properties. ${ }^{3}$ $\mathrm{CBD}$ is not psychoactive but exhibits similar beneficial effects as THC. $^{4}$

The absence of psychoactive effects and the abundance of anecdotal claims of the effectiveness of CBD across a wide variety of conditions in both mainstream and social media have created significant public interest in CBD products, typically as oils. Such interest has led to increased consumer availability. Generally, commercially available CBD oils mainly consist of $\mathrm{CBD}$ and carrier oil and may contain flavorings, terpenes, or other cannabinoids like CBDA or trace amounts of THCA and THC. ${ }^{5}$ Incorrect or misleading labels for the cannabinoid content of CBD products and indiscriminate use of CBD may lead to various issues. The presence of THC in commercial CBD oils can occur because of the fact that even CBD-rich varieties of cannabis produce a small amount of THC. ${ }^{6}$
Therefore, naturally derived CBD extracts may contain some THC in the final products, and oil consumers could thus be taking THC without knowing so. Additionally, if the THC content of a CBD product exceeds the maximally allowed limit, this could lead to legal problems. Many nations stipulate a zero-tolerance policy or a maximum THC level (0.05-1\%) for CBD products. 6 ,7

As THC and CBD are structural isomers (accurate molecular weight of $314.2246 \mathrm{Da}$ ), differentiating as well as quantifying THC and CBD is challenging. Generally, gas chromatography (GC)- or liquid chromatography (LC)-based techniques are employed, and typically run times of 10-20 min are required to achieve baseline separation. ${ }^{8}$ Additionally, for GC-based techniques, the detection of the acidic precursors THCA and CBDA (accurate molecular weight of 358.2144 Da) is not feasible without derivatization. ${ }^{9}$

Received: October 10, 2020

Accepted: February 2, 2021

Published: February 12, 2021 
Simple and rapid methods to screen THC and CBD have been developed, including electrochemical ${ }^{2}$ and colorimetric $^{10,11}$ approaches. However, these methods cannot differentiate cannabinolic acids (THCA and CBDA) from the respective cannabinol forms (THC and $\mathrm{CBD})^{2}$ or distinguish THC and $\mathrm{CBD} ;{ }^{11}$ moreover, these methods cannot achieve quantitative results. ${ }^{10}$

Even with mass spectrometry (MS), it is difficult to differentiate between THC and CBD as they have the same mass and give identical collision-induced dissociation (CID) fragmentation patterns in positive ionization mode. ${ }^{12}$ In negative mode, it has been reported that there may exist a unique THC MS/MS fragment $(m / z 191)$ under specific conditions, which could allow the differentiation of these two compounds to some extent. ${ }^{13}$ However, in another report, this fragment-under different conditions-has also been demonstrated for CBD. ${ }^{14}$ Therefore, only relying on this fragment for distinguishing between the isomers and their quantification remains challenging.

To enhance MS performance and improve ionization efficiency, adduct formation with $\mathrm{Ag}(\mathrm{I})$ ions has been exploited in ion mobility spectrometry, ${ }^{15}$ electrospray ionization mass spectrometry (ESI-MS), ${ }^{16-18}$ secondary ion mass spectrometry, ${ }^{19}$ and matrix-assisted laser desorption ionization mass spectrometry. ${ }^{20} \mathrm{Ag}(\mathrm{I})$ coordinates strongly with alkenes and weakly with polar groups like hydroxyls. The presence of multiple isolated $\mathrm{C}=\mathrm{C}$ bonds, especially 1,5 -dienes, strongly increases complexation, which is used in the analysis of certain olefinic compounds. ${ }^{21-23}$ However, to the best of our knowledge, $\operatorname{Ag}(\mathrm{I})$ adduct formation has been rarely applied to ambient ionization mass spectrometry (AIMS) ${ }^{24}$ with the notable exception of improving the detection of olefins with desorption electrospray ionization (DESI)-MS. ${ }^{25}$

Paper spray ionization mass spectrometry (PS-MS), first reported in 2010, ${ }^{26}$ is an AIMS method, well known for its fast analysis at low cost of consumables. ${ }^{27}$ In PS-MS, analytes are ionized from a paper tip by application of a solvent and an electric field, and thus it is one of the many variants of electrospray ionization. ${ }^{28}$ Importantly, paper can be readily modified to enhance PS-MS selectivity and sensitivity through surface modification or coating. ${ }^{27-30}$

In this work, we investigated the combination of $\operatorname{Ag}(\mathrm{I})$ ions and PS-MS for the rapid and selective analysis of THC and $\mathrm{CBD}$, in particular for detecting low concentrations of THC in CBD oils. Based on the different complexations of THC (weak; single alkene $\mathrm{C}=\mathrm{C}$ bond) and $\mathrm{CBD}$ (strong; 1,5-diene moiety) with $\mathrm{Ag}(\mathrm{I})$ ions, ${ }^{21}$ we hypothesized that the formed THC and CBD $\mathrm{Ag}(\mathrm{I})$ adducts will exhibit different stabilities. In turn, this could lead to different fragmentation patterns in tandem MS. If so, simple and fast differentiation of THC and $\mathrm{CBD}$ as well as semiquantitative analysis should be achievable. As hypothesized, the quasi-molecular $\mathrm{Ag}(\mathrm{I})$ adducts of THC $[\mathrm{THC}+\mathrm{Ag}]^{+}$and $\mathrm{CBD}[\mathrm{CBD}+\mathrm{Ag}]^{+}$at $\mathrm{m} / z 421$ and 423 give different product ions in tandem MS, at $m / z 313$ for THC and $m / z 353$ and 355 for CBD. These product ions were then used to distinguish between THC and CBD and to determine their ratio within a few minutes in commercial CBD oils.

\section{MATERIALS AND METHODS}

Chemicals and Reagents. Acetonitrile, methanol, and tert-butyl methyl ether (MTBE; HPLC-grade) were purchased from Biosolve Chimie SARL (Dieuze, France). Formic acid (HPLC-grade) and silver nitrate (analytical grade) were purchased from Fisher Scientific (Loughborough, Leicestershire). Deionized water was obtained from a Milli-Q Direct ultrapure water system (Millipore, USA). THC and CBD standards were obtained from cannabis flowers and CBD oil, respectively. According to nuclear magnetic resonance (NMR), thin layer chromatography (TLC), and UHPLC-UV data (Supporting Information, Figures S1-S3), their purity was $>98 \%$. Chromatography paper was purchased from Hangzhou Special Paper Co., Ltd. (Hangzhou, China). Different brands of pure CBD oils were purchased from health shops (Wageningen, the Netherlands) or ordered online. Information on all CBD oils is shown in the Supporting Information, Table S1. Ammonium acetate (analytical grade) was obtained from Merck (Darmstadt, Germany). Santonin (analytical grade) was purchased from Sigma (St. Louis, MO, USA).

Preparation of Paper Substrate. Chromatography paper was cut into isosceles triangles with a height of $10 \mathrm{~mm}$ and a base of $5 \mathrm{~mm}$, employing a paper cutter made in-house. These paper tips were put in a bottle with methanol and washed for $30 \mathrm{~min}$ in an ultrasonic bath at room temperature. Afterward, methanol was decanted, and the bottle with tips was placed in a fume hood for $30 \mathrm{~min}$ at room temperature and then dried at $60{ }^{\circ} \mathrm{C}$ in a vacuum oven for $12 \mathrm{~h}$. The resulting paper tips are referred to as clean.

Preparation of $\mathbf{A g}(\mathrm{I})$-Impregnated Paper Substrate. For the preparation of $\mathrm{Ag}(\mathrm{I})$-impregnated tips, $60 \mathrm{~mL}$ of ultrapure water was added to $1.02 \mathrm{~g}$ silver nitrate in a $125 \mathrm{~mL}$ wide-mouth brown bottle, which was placed in an ultrasonic bath for 2 min to obtain a $0.10 \mathrm{~mol} \mathrm{~L}^{-1} \mathrm{AgNO}_{3}$ solution. Clean paper tips were immersed in the $\mathrm{AgNO}_{3}$ solution, and after capping, the bottle was placed in an ultrasonic bath. After 15 min of sonication, water in the ultrasound bath was replaced because otherwise the temperature of water would become too high ( should not be above ca. $40{ }^{\circ} \mathrm{C}$ ), making the surface of the paper rough or even damaging the tips. After another 15 min, the $\mathrm{AgNO}_{3}$ solution was decanted, and the bottle without cap was heated in an oven at $100{ }^{\circ} \mathrm{C}$ for at least $1 \mathrm{~h}$ to evaporate most water. Finally, the tips were taken out of the bottle and dried in a vacuum oven at $60{ }^{\circ} \mathrm{C}$ for $12 \mathrm{~h}$. $\mathrm{Ag}(\mathrm{I})$ impregnated paper was put in tin foil and stored in a desiccator away from light. Clean paper tips were used for PS-MS, and the $\mathrm{Ag}(\mathrm{I})$-impregnated paper tips were used for $\mathrm{Ag}(\mathrm{I})$-impregnated paper spray MS (AgPS-MS).

Paper Spray Setup. The paper tip was positioned by an alligator clip, which was part of a modified DESI ion source (Prosolia, USA) equipped with a rotational and $x-y-z$ positioner, and was directly connected to the HV supply of the ion source. The front of the paper tip was pointing toward the MS inlet at 4-6 mm. A sample solution of $15 \mu \mathrm{L}$ was added using an Eppendorf pipette $(10-100 \mu \mathrm{L})$. After this, a voltage of $4 \mathrm{kV}$ was applied. The paper spray ion source was connected to either a high-resolution MS or to a linear ion trap MS.

Linear Ion Trap Mass Spectrometer. A Thermo LXQ linear ion trap mass spectrometer (Thermo Fisher Scientific, San Jose, CA, USA) was used in positive mode, with a capillary voltage of $49 \mathrm{~V}$, tube lens of $85 \mathrm{~V}$, and a capillary temperature of $350{ }^{\circ} \mathrm{C}$, unless indicated otherwise. All full-scan measurements were performed with a scan range of $\mathrm{m} / z$ 100.0-2000.0. For all $\mathrm{MS}^{n}$ fragmentation measurements, CID energy was determined as the energy at which the target product ions had the highest abundance while the precursor ion or ions had not 
yet disappeared completely. The isolation width was set to include all desired target precursor ions.

Quadrupole Orbitrap High-Resolution Mass Spectrometer. For accurate mass measurements, the paper spray device was coupled to a Q-Exactive quadrupole orbitrap highresolution MS (Thermo Fischer Scientific). All measurements were performed in positive mode with a mass resolution of $140,000 \mathrm{fwhm}$ and a maximum injection time of $100 \mathrm{~ms}$. The capillary temperature was $350{ }^{\circ} \mathrm{C}$, and the S-lens RF level was 47. All full-scan measurements were performed with a scan range of $\mathrm{m} / z$ 100.0-2000.0. For molecular formula confirmation of the main peaks in $\mathrm{MS}^{1}$ and $\mathrm{MS}^{2}$ spectra, full-scan and CID fragmentation scan modes were used, respectively. For the determination of the molecular formula of the main peaks in $\mathrm{MS}^{3}$ and $\mathrm{MS}^{4}$ spectra from the LXQ analysis, insource fragmentation and CID fragmentation were combined to provide higher energies for further fragmentation. Thermo Scientific Xcalibur 2.2 software was used for data acquisition and processing. The intensity of ions with $m / z$ values within $\pm 5 \mathrm{ppm}$ of the theoretical $\mathrm{m} / z$ is shown in the extracted ion chromatogram (EIC).

Ag(I) Complexation (Argentation) ChromatographyMass Spectrometry. Following a previously described method, ${ }^{21}$ a strong cation exchange HPLC column (Nucleosil SA, $100 \mathrm{~A}, 5 \mu \mathrm{m}, 2.1 \times 250 \mathrm{~mm}$; Grace) was flushed with an aqueous $1 \% \mathrm{NH}_{4} \mathrm{OAc}$ solution at $0.50 \mathrm{~mL} \mathrm{~min}^{-1}$ for $1 \mathrm{~h}$, followed by distilled water for $1 \mathrm{~h}$. An aqueous $\mathrm{AgNO}_{3}$ solution $(0.20 \mathrm{~g} / \mathrm{mL})$ was injected onto the column via an autosampler in $50 \mu \mathrm{L}$ aliquots at 1 min intervals; 20 min after the last injection, the column was washed with $\mathrm{MeOH}$ for $1 \mathrm{~h}$.

A 1220 Infinity II LC system (Agilent Technologies, Santa Clara, USA) was coupled to the LXQMS. Separation of THC and $\mathrm{CBD}$ was achieved using the loaded $\mathrm{Ag}(\mathrm{I})$ column, with $\mathrm{MeOH}$ as the mobile phase, at a flow rate of $0.80 \mathrm{~mL} \cdot \mathrm{min}^{-1}$. The complexes of eluted compounds were directed into the LXQ MS and analyzed under full-scan or product ion scan mode. The LXQ settings were identical to those described for the PS-MS measurements except for the sheath gas flow rate of 15 (arbitrary units).

UHPLC-UV Analysis. A Zorbax Eclipse Plus C18 column $(2.1 \mathrm{~mm} \times 50 \mathrm{~mm}, 1.8 \mu \mathrm{m}$; Agilent Technologies, Santa Clara, CA, USA) was coupled to a 1290 Infinity ultra-high performance liquid chromatography (UHPLC) system (Agilent Technologies, Santa Clara, USA), with a diode array detector. The mobile phase consisted of $5 \mathrm{mM}$ formic acid in both water (mobile phase A) and acetonitrile (mobile phase $\mathrm{B}$ ), and the flow rate was $0.80 \mathrm{~mL} \cdot \mathrm{min}^{-1}$. Isocratic elution for 2 min with $25 \%$ B was followed by a gradient toward $100 \%$ B in $7 \mathrm{~min}$. After being in this condition for $4 \mathrm{~min}$, the system was returned to $25 \% \mathrm{~B}$ in $1 \mathrm{~min}$ and then re-equilibrated for $3 \mathrm{~min}$ at $25 \% \mathrm{~B}^{31}$

Sample Preparation and Extraction. Stock solutions of THC and CBD were prepared in $\mathrm{MeOH}$ at $1.00 \mathrm{mg} \cdot \mathrm{mL}^{-1}$. Samples for the determination of recovery, matrix effects, and calibration curves were constructed by spiking sunflower oil with THC or CBD stock solutions (see following sections). For extraction, $50.0 \mathrm{mg}$ of spiked or blank oil was precisely weighed and $2.00 \mathrm{~mL}$ of $\mathrm{MeOH}$ was added. The extraction was performed by stirring samples with a magnetic stirring bar and a magnetic stirrer (IKA Labortechnic, IKAMAG RCT basic, Germany) for $1,5,10,20$, or $30 \mathrm{~min}$. Afterward, $1.00 \mathrm{~mL}$ of the supernatant of each extracted sample was collected for analysis.
Commercial CBD oil samples were diluted with sunflower oil to $0.2 \%(\mathrm{w} / \mathrm{w} \%) \mathrm{CBD}$ in oil based on the labeled concentration. A magnetic stirring bar was used to stir the oil vigorously for $1 \mathrm{~min}$. A $50.0 \mathrm{mg}$ of the diluted CBD oil was precisely weighed for the following analysis. A $2.00 \mathrm{~mL}$ of $\mathrm{MeOH}$ was added to $50.0 \mathrm{mg}$ of the diluted CBD oil for extraction. The extraction procedure was performed in triplicate by stirring these samples with a magnetic stirring bar for $1 \mathrm{~min}$. A volume of $1.00 \mathrm{~mL}$ of the supernatant of each extracted sample was taken for further MS or UHPLC analysis.

Determination of Recovery and Matrix Effects. Extraction recovery and matrix effects were determined according to the procedure by Gottardo et al., ${ }^{32}$ with minor modifications, as described below (see also Supporting Information, Table S2).

Sunflower oil samples containing $0.20 \%$ THC (THC/oil, w/ w \%) and $0.20 \% \mathrm{CBD}(\mathrm{CBD} / \mathrm{oil}, \mathrm{w} / \mathrm{w} \%)$ were extracted with $\mathrm{MeOH}$ for $1,5,10,20$, and $30 \mathrm{~min}$ (sample type III, $n=3$ ), whereas, for comparison, analogous extractions of the blank matrix (sunflower oil) were performed. These blank matrix extracts were then spiked with the same amount of THC and CBD per $\mathrm{mL}$ of methanol (sample type II, $n=3$ ). The final methanolic solutions were analyzed by the UHPLC-UV method, and the recovery was calculated as the ratio of the averaged peak areas from set III to set II, expressed as a percentage $($ recovery $(\%)=\mathrm{III} / \mathrm{II} \times 100)$ for both $\mathrm{THC}$ and CBD.

For the matrix effect measurements, the same amounts of THC and CBD were spiked in the blank matrix extract (sample type II, $n=3$ ) or pure $\mathrm{MeOH}$ (sample type I, $n=3$ ). These methanolic solutions were analyzed by AgPS-MS. The EIC was normalized to the total ion chromatogram (TIC) to correct for the spray instability and irreproducibility (EIC/ TIC). The matrix effect was calculated as the ratio of the THC $\mathrm{MS}^{2}$ characteristic signal $(\mathrm{m} / z$ 313) from set II to set I, subtracted by 1 , and expressed as a percentage (matrix effect $(\%)=(\mathrm{II} / \mathrm{I}-1) \times 100)$. The matrix effect of CBD $(\mathrm{m} / z 353$ $+355)$ was measured and calculated with the same methods as that for THC. A negative matrix effect percentage represents signal suppression and a positive percentage represents signal enhancement.

Calibration Curve Construction and Evaluation of Accuracy, Precision, LOD, and LOQ. THC and CBD stock solutions were used to prepare oil samples with different THC/CBD ratios (0.001, 0.002, 0.005, 0.01, 0.02, 0.05, 0.1, $0.2,0.5$, and $1 ; n=3$ per ratio), keeping the CBD content constant at $0.20 \%(\mathrm{CBD} / \mathrm{oil}, \mathrm{w} / \mathrm{w} \%)$. To achieve this, appropriate volumes of methanolic stock solutions were mixed and dried under nitrogen and then reconstituted in $50.0 \mathrm{mg}$ sunflower oil. These samples were extracted as described above, and the methanolic extract was analyzed with UHPLCUV and AgPS-MS methods. The peak area ratios of THC/ $\mathrm{CBD}$ at $215 \mathrm{~nm}$ or the characteristic $\mathrm{MS}^{2} \mathrm{EIC}$ area ratios of $\mathrm{THC} / \mathrm{CBD}$ were plotted against the concentration ratios of THC/CBD for constructing the calibration curves for UHPLC-UV detection and AgPS-MS, respectively.

The accuracy and precision of the method were evaluated at three THC/CBD ratios (low (0.004), medium (0.07), and high (0.3) in sunflower oil ( $n=3$ per ratio). The preparation of these three samples was identical to the preparation of calibration curve samples. Precision was calculated as the relative standard deviation (RSD \%) $(n=3)$. Accuracy was 
calculated as the relative deviation (\%) of the calculated mean value from the respective reference value.

Santonin with an accurate molecular weight of 246.1256 Da was used as the internal standard for the determination of the absolute LOD and LOQ of the AgPS-MS method for THC. The characteristic $\mathrm{MS}^{2}$ peaks are $\mathrm{m} / z 309$ and 311 from their precursor ions, $m / z 353$ and 355, when analyzed by AgPS-MS. A $100.0 \mu \mathrm{L}$ of $300.0 \mu \mathrm{g} \cdot \mathrm{mL}^{-1}$ santonin in $\mathrm{MeOH}$ was mixed with $0.0,1.0,2.5,5.0,10.0,25,50$, or $100 \mu \mathrm{L}$ of a $200.0 \mu \mathrm{g}$. $\mathrm{mL}^{-1}$ THC solution in $\mathrm{MeOH}$, and $\mathrm{MeOH}$ was added to 1.00 $\mathrm{mL}$ to prepare $0.0,0.2,0.5,1.0,2.0,5.0,10.0$, or $20.0 \mu \mathrm{g} \cdot \mathrm{mL}^{-1}$ THC solutions, with $30 \mu \mathrm{g} \cdot \mathrm{mL}^{-1}$ santonin $(n=3$ per concentration). The samples were analyzed by AgPS-MS under the same conditions as those used for the THC/CBD ratio analysis. The characteristic $\mathrm{MS}^{2}$ fragment EIC area ratios of THC $(m / z 313)$ to santonin $(m / z 309)$ were plotted against the concentrations of THC for constructing a curve. LOD and LOQ were calculated as follows: LOD $=3 \times \mathrm{SD}$ of blank/ slope of the curve; LOQ $=10 \times \mathrm{SD}$ of blank/slope of the curve.

Quantum Chemical Computations. Calculations of the binding energies of $\mathrm{THC}$ and $\mathrm{CBD}$ toward $\mathrm{Ag}(\mathrm{I})$ were performed with the Gaussian 16 suite of programs, with the B3LYP and wB97XD functionals as implemented in there; a 6$311+\mathrm{G}(\mathrm{d}, \mathrm{p})$ basis set was used throughout. The electrostatic potential map was generated using Gaussview 6 .

\section{RESULTS AND DISCUSSION}

THC and CBD are isomers with an accurate molecular weight of $314.2246 \mathrm{Da}$. It is difficult to distinguish between these two species by AIMS, ${ }^{31,33,34}$ including MS/MS analysis, as both compounds fragment into product ions with the same mass. When analyzed by PS-MS in (+) mode and setting $m / z 315$ as the precursor ion with an isolation width of 1.5, THC (Figure $1 \mathrm{~A}$ ) and $\mathrm{CBD}$ (Figure 1B) indeed yield the same $\mathrm{MS}^{2}$ spectrum with the main fragments of $m / z 193$ and $m / z 259$, making differentiation impossible.

In order to effectively combine the advantages of AIMS and the potential of $\operatorname{Ag}(\mathrm{I})$ ions to bind differently to different olefinic compounds, $\mathrm{Ag}(\mathrm{I})$ was combined with PS-MS by using an $\mathrm{Ag}(\mathrm{I})$-impregnated paper. Quasi-molecular species at $\mathrm{m} / \mathrm{z}$ 421 and 423 were observed for both THC and CBD because of the existence of two silver isotopes, ${ }^{107} \mathrm{Ag}$ (52\%) and ${ }^{109} \mathrm{Ag}$ (48\%). By setting $m / z 422$ as the precursor ion and using an isolation width of 4 (thus including both $\mathrm{m} / z 421$ and 423), different fragmentation patterns for THC (Figure 1C) and CBD (Figure 1D) were observed. Specifically, there was only one product ion at $m / z 313$ for THC, and for CBD, the most pronounced fragments appeared at $m / z 353$ and 355, which is the same fragment, with the mass difference because of two silver isotopes. The accurate masses and molecular formulas of the characteristic peaks for both THC and CBD $\mathrm{Ag}(\mathrm{I})$ adducts, as well as their fragments, were determined with MS/ HRMS (Figure 2). After CID, $[\mathrm{THC}+\mathrm{Ag}]^{+}$loses its $\mathrm{Ag}(\mathrm{I})$ during the $\mathrm{MS}^{2}$ stage, whereas the majority of $[\mathrm{CBD}+\mathrm{Ag}]^{+}$ retains $\mathrm{Ag}(\mathrm{I})$ until the $\mathrm{MS}^{3}$ stage (Supporting Information, Figure S4). This finding suggests-as hypothesized-that $\mathrm{Ag}(\mathrm{I})$ binds more strongly to CBD than to THC and that this indeed leads to substantial differences in CID patterns.

This hypothesis was further substantiated by argentation HPLC-MS/MS results (Supporting Information, Figure S5). CBD [capacity factor $\left(k^{\prime}\right)=11.2$ ] eluted much later than THC $\left(k^{\prime}=0.2\right)$, which means that $\mathrm{CBD}$ has a much stronger retention than $\mathrm{THC}$ on the $\mathrm{Ag}(\mathrm{I})$ column. Moreover, by quantum chemical wB97XD/6-311+G(d,p) simulations, the $\mathrm{CBD}+\operatorname{Ag}(\mathrm{I})$ complex was found to be $\sim 12 \mathrm{kcal} / \mathrm{mol}$ more stable than the $\mathrm{THC}+\mathrm{Ag}(\mathrm{I})$ complex (Supporting Information, Figure S6), which means that a higher energy is required for the $\mathrm{CBD}+\mathrm{Ag}(\mathrm{I})$ complex to lose its $\mathrm{Ag}(\mathrm{I})$ compared to the $\mathrm{THC}+\mathrm{Ag}(\mathrm{I})$ complex.

Based on the above findings, a mechanism for the MS fragmentation of THC and CBD in the presence of $\mathrm{Ag}(\mathrm{I})$ was proposed. As shown in Figure 2, the $[\mathrm{THC}+\mathrm{Ag}]^{+}$adducts lose $\mathrm{AgH}$ during the $\mathrm{MS}^{2}$ stage, but the majority of $[\mathrm{CBD}+$ $\mathrm{Ag}]^{+}$adducts lose $\mathrm{C}_{5} \mathrm{H}_{8}$ during the $\mathrm{MS}^{2}$ stage, most likely the neutral loss of 2-methyl-1,3-butadiene. The $\mathrm{Ag}(\mathrm{I})$ ion of [CBD $+\mathrm{Ag}]^{+}$adducts is lost from $\mathrm{CBD}$ as $\mathrm{AgCH}_{3}$ during the $\mathrm{MS}^{3}$ stage (Supporting Information, Figure S4). This difference reflects the much stronger binding of $\mathrm{Ag}(\mathrm{I})$ to $\mathrm{CBD}$ than to THC, which is attributed to the 1,5-diene system of CBD. ${ }^{21-23}$ The loss of the $\operatorname{Ag}(\mathrm{I})$ ion during the $\mathrm{MS}^{3}$ stage can be explained too because in the $m / z 353 / 355$ CBD fragment, $\operatorname{Ag}(\mathrm{I})$ complexes with a 1,3 -diene system. This complexation is weaker than that with the 1,5-diene system in the quasimolecular ion $(m / z 421$ and $m / z 423)$ of $\mathrm{CBD}^{21}$ In the case of the $[\mathrm{THC}+\mathrm{Ag}]^{+}$adduct, because of the oxygen-gemdimethyl carbon bond in THC, methyl-butadiene cannot be split off, and the loss of the only weakly bound $\mathrm{Ag}(\mathrm{I})$ is to be expected. The neutral loss of either $\mathrm{AgH}$ or $\mathrm{AgCH}_{3}$ is supported by the literature. $^{35,36}$

AgPS-MS/MS can clearly be employed to distinguish THC from CBD. However, in the $\mathrm{MS}^{2}$ spectrum of CBD (Figure $1 \mathrm{D})$, there is also a minor peak at $m / z 313$, which equals the mass of the characteristic MS ${ }^{2}$ fragment of THC. This finding complicates the determination of the THC/CBD ratio. If this $\mathrm{m} / z 313$ fragment would originate from a CBD-to-THC conversion, and would therefore be variable, this would preclude an accurate determination of the THC/CBD ratio. However, if it is a product ion directly originating from the fragmentation of $\mathrm{CBD}$, its intensity can potentially be corrected for.

To elucidate whether the $m / z 313$ fragment in the CBD spectrum originated from $\mathrm{CBD}$ or $\mathrm{THC}$, further fragmentation analysis was carried out for THC and CBD. The THC $\mathrm{m} / z$ 313 fragment yielded the main fragment at $\mathrm{m} / z 217$ with some other minor fragments during the $\mathrm{MS}^{3}$ stage (Supporting Information, Figure S7). For CBD, the fragmentation of $\mathrm{m} / z$ 313 yielded a similar $\mathrm{MS}^{3}$ spectrum in which the peak at $\mathrm{m} / z$ 243 reproducibly had double relative intensity compared to the THC spectrum. For the fragments from $\mathrm{m} / z 313$ of THC, $\mathrm{m} / z$ 243 and $m / z 245$ had equal abundance. For the fragments from $\mathrm{m} / z 313$ of CBD, the abundance of $\mathrm{m} / z 243$ almost doubled that of $m / z 245$. This suggests that the fragment of $m / z 313$ in the CBD $\mathrm{MS}^{3}$ spectrum is structurally different from the $m / z 313$ fragment in the THC MS 3 spectrum.

To further exclude the possibility of conversion from CBD to THC, the peak area ratio of $m / z 313 /(353+355)$ was measured for pure CBD under different MS parameters $(n=3$ per parameter), namely various source temperatures, CID energies, and CBD concentrations, as well as on two different mass spectrometers. The ratio was always constant within 2.7$3.0 \%$, regardless of the parameter changes (Supporting Information, Figure S8), strongly suggesting that the fragment at $m / z 313$ in the $\mathrm{MS}^{2} \mathrm{CBD}$ spectrum is a genuine fragment of $\mathrm{CBD}$, and not due to in situ conversion to THC, as that would have likely led to a change in the $m / z 313 /(353+355)$ ratio 

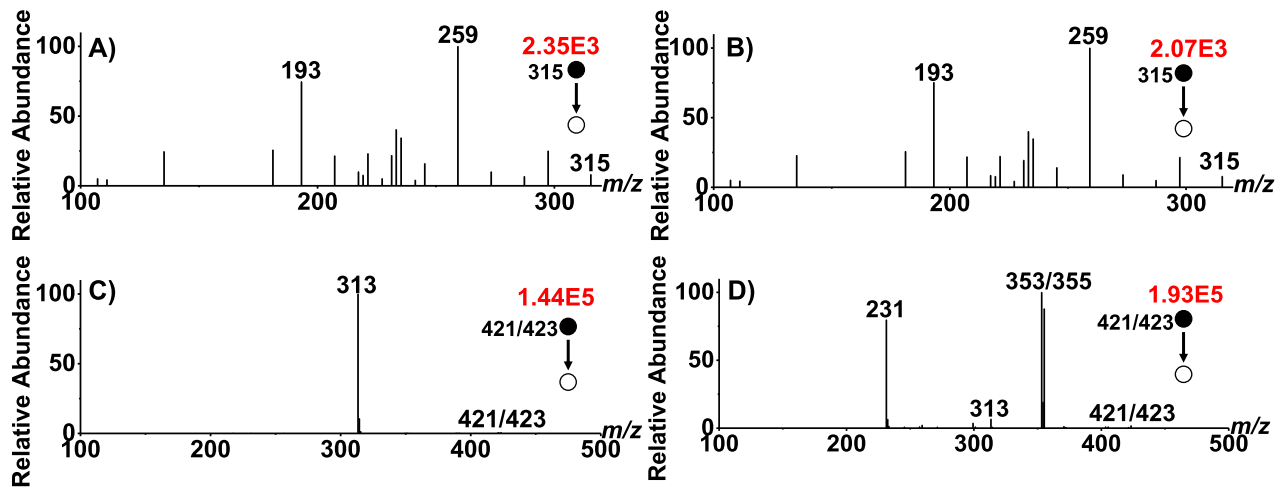

Figure 1. PS-MS ${ }^{2}$ spectrum of THC (A), PS-MS ${ }^{2}$ spectrum of CBD (B), AgPS-MS ${ }^{2}$ spectrum of THC (C), and AgPS-MS ${ }^{2}$ spectrum of CBD (D).

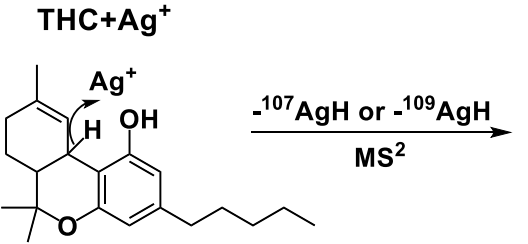

$\left[\mathrm{C}_{21} \mathrm{H}_{30} \mathrm{O}_{2}{ }^{107} \mathrm{Ag}\right]^{+}$:

Theoretical $M W=421.1291$

Measured $M W=421.1281$

$\left[\mathrm{C}_{21} \mathrm{H}_{30} \mathrm{O}_{2}{ }^{109} \mathrm{Ag}\right]^{+}$:

Theoretical $M W=423.1288$

Measured $M W=423.1279$

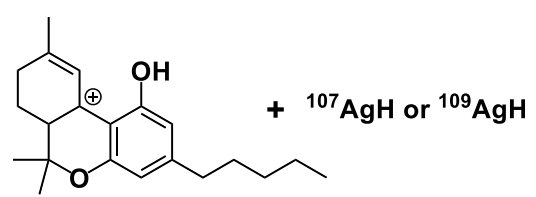

$\left[\mathrm{C}_{21} \mathrm{H}_{29} \mathrm{O}_{2}\right]^{+}$:

Theoretical $M W=313.2162$

Measured $M W=313.2156$

another 6 resonance structures, e.g.,<smiles>CCCCCc1cc(O)c2c(c1)OC(C)(C)C1C=C2C=C(C)CC1</smiles>

$\mathrm{CBD}+\mathrm{Ag}^{+}$

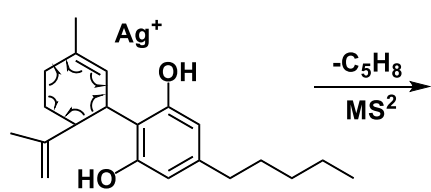<smiles>C=CC(=C)C</smiles>

$\left[\mathrm{C}_{21} \mathrm{H}_{30} \mathrm{O}_{2}{ }^{107} \mathrm{Ag}\right]^{+}$:

Theoretical $M W=421.1291$

Measured $M W=421.1297$

$\left[\mathrm{C}_{21} \mathrm{H}_{30} \mathrm{O}_{2}{ }^{109} \mathrm{Ag}\right]^{+}$:

Theoretical $M W=423.1288$

Measured $M W=423.1291$

$\left[\mathrm{C}_{16} \mathrm{H}_{22} \mathrm{O}_{2}{ }^{107} \mathrm{Ag}\right]^{+}$:

Theoretical $M W=353.0665$

Measured $M W=353.0676$

$\left[\mathrm{C}_{16} \mathrm{H}_{22} \mathrm{O}_{2}{ }^{109} \mathrm{Ag}\right]^{+}$:

Theoretical $M W=355.0662$

Measured $M W=355.0663$

Figure 2. Proposed mechanism for $\mathrm{MS}^{2}$ fragmentation of THC and $\mathrm{CBD}$ in the presence of $\mathrm{Ag}(\mathrm{I})$.

with a change in any of these parameters. Taking all the above into consideration, we argue that the $m / z 313$ signal in the CBD AgPS-MS ${ }^{2}$ spectrum is derived from CBD itself and not from THC.

When keeping all equipment parameters unchanged, the EIC area ratio of $m / z 313$ to $(m / z 353+m / z 355)$ in the CBD $\mathrm{MS}^{2}$ spectrum is constant $(0.028 \pm 0.001)$ for a range of $\mathrm{CBD}$ concentrations $(0.5-1000 \mathrm{ppm})$. As a result, the $m / z 313$ signal resulting from the $\mathrm{CBD}$ spectrum can, in the MS data from samples, be subtracted as the background value. Quantitative analysis of the THC/CBD ratio in samples can thus be achieved by measuring in the MS ${ }^{2}$ spectra the EIC area ratio of $m / z 313$ to $(m / z 353+m / z 355)$ and then subtracting the background value of $0.028 \pm 0.001$ from pure CBD.
An important consideration for the cannabinoid analysis is that cannabinoids from plants are effectively in a "prodrug" form, existing as cannabinolic acids that must be decarboxylated to their respective cannabinol form to have pharmacological effects. ${ }^{2}$ This decarboxylation, for example, occurs while smoking; however, upon oral consumption, no CBDA or THCA present is converted to CBD or THC by enzymatic or other processes. ${ }^{37,38}$ If the production and processing of $\mathrm{CBD}$ oils does not remove all THCA and CBDA, some THCA and CBDA might still be present in the final CBD oil products. To evaluate whether THCA and CBDA would decarboxylate to their respective cannabinol forms during the AgPS-MS analysis and would thus interfere with the quantification of the THC/ CBD ratio, standard solutions of THCA and CBDA were analyzed with AgPS-MS (Supporting Information, Figure S9), 
UHPLC-UV (Supporting Information, Figure S10), and HRMS (Supporting Information, Figures S11 and 12). Both THCA and CBDA formed quasi-molecular ions (Ag(I) adducts) at $m / z 465$ and 467 , and no fragments at $m / z 421$ and 423 can be observed. Therefore, any CBDA or THCA present does not interfere with the analysis of THC and CBD when choosing $\mathrm{m} / \mathrm{z} 421 / 423$ as the precursor ions for fragmentation to obtain the characteristic $\mathrm{MS}^{2}$ fragments of THC and CBD.

Recovery and Matrix Effect. To apply the findings in the practical analysis of THC and CBD in CBD oil samples, an analytical method consisting of an easy sample pretreatment step and a fast AgPS-MS procedure was developed. To determine the recovery and matrix effect, sunflower oil was selected as the matrix for THC and CBD because of the similar fatty acid composition ${ }^{39}$ as hempseed oil (the main constituent of commercial CBD oil). Additionally, THC and CBD do not occur in sunflower oil.

The recovery was calculated by comparing the extract of a spiked sample to the extract of a blank matrix sample that was spiked with the same amount after extraction. In both cases, the co-extracted matrix compounds will be the same in the extract, and the only difference is caused by the recovery of the analytes. These samples were analyzed by the UHPLC-UV method, and the extraction recovery was calculated as the ratio of the average UHPLC chromatogram THC or CBD peak areas from extracted samples and nonextracted samples, and expressed as a percentage (Figure 3A). Different extraction
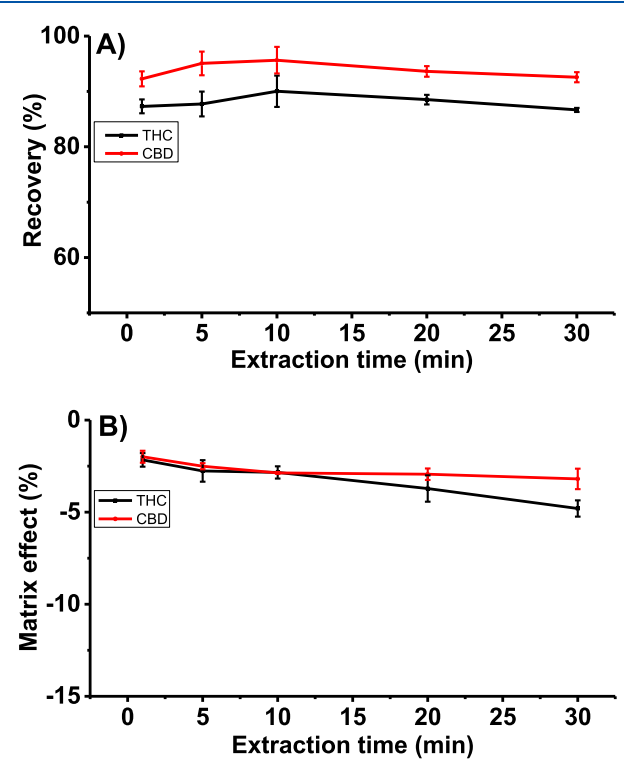

Figure 3. Recovery (A) and matrix effect (B) for THC and CBD analysis after extraction $v s$ the extraction times. Error bars represent standard deviation $(n=3)$. times have a limited effect on the recovery of both THC (86.7-90.0\%) and CBD (92.3-95.6\%). The slightly lower recovery of THC can be explained by the fact that THC is less polar than $\mathrm{CBD}$ and more likely to remain in the nonpolar sunflower oil.

The matrix effect was assessed by comparing spiked methanol with a spiked matrix extract. In both solutions, THC and CBD do not undergo any extraction step, that is, the recovery is $100 \%$. However, pure $\mathrm{MeOH}$ does not contain extracted matrix compounds, whereas the blank matrix sample does. Matrix compounds might interfere with the analysis through ion suppression. The calculated ME for AgPS-MS from sunflower oil is shown in Figure 3B for various extraction times $(-2.2$ to -4.8 for $\mathrm{THC}$ and -2.0 to -3.2 for CBD). Both THC and CBD suffer the least from ion suppression when the extraction time is $1 \mathrm{~min}(-2.2 \pm 0.4 \%$ for THC and $-2.0 \pm 0.3 \%$ for $\mathrm{CBD}$ ), and the ion suppression becomes more pronounced when extending the extraction time (Figure 3B). However, in view of the tolerant limit for matrix effects $( \pm 25 \%),{ }^{40}$ longer extraction times would also be possible if desired.

To correct for the extraction efficiency and matrix effects in the quantification, a matrix-based calibration curve was constructed. As extraction for $1 \mathrm{~min}$ resulted in acceptable recoveries for THC and CBD $(87.3 \pm 1.2 \%$ for THC and 92.3 $\pm 1.4 \%$ for CBD $)$, and minimal matrix effects $(-2.2 \pm 0.4 \%$ for $\mathrm{THC},-2.0 \pm 0.3 \%$ for $\mathrm{CBD}$ ) as well as high time efficiencies, the $1 \mathrm{~min}$ extraction time was used in all subsequent experiments.

Calibration Curve, Precision, and Accuracy. An AgPSMS calibration curve was constructed relating the characteristic $\mathrm{MS}^{2} \mathrm{EIC}$ area ratio of THC/CBD to the concentration ratio of THC/CBD in samples (Supporting Information, Figure S13A). The $y$-intercept of this curve is 0.0280 , which means that when the sample does not contain THC, the characteristic signal ratio of THC to CBD is 0.0280 , consistent with the formation of the minor fragment at $\mathrm{m} / z 313$ purely by CBD. For comparison, a UHPLC-UV curve was established associating the characteristic peak area ratio of $\mathrm{THC} / \mathrm{CBD}$ with the concentration ratio of THC/CBD in samples. There is good linearity $\left(R^{2}=0.9896\right)$ of the AgPS-MS signal intensity over the full working range of THC/CBD ratios from 0.001 to 1 (Supporting Information, Figure S13). Even though the UHPLC-UV curve has a better linearity $\left(R^{2}=0.9998\right)$, each run takes $17 \mathrm{~min}$, which is much longer than that of an AgPSMS experiment (<30 s).

Accuracy and precision of the methods were evaluated at three THC/CBD ratios of $0.004,0.07$, and 0.3 (low, medium, and high levels) in spiked sunflower oil samples and are shown in Table 1. The AgPS-MS method has comparable analytical performance as the UHPLC-UV method, with accuracy and precision for medium and high concentrations as well as acceptable accuracy and precision at low THC concentrations.

Table 1. Precision (\% RSD) and Accuracy (\% Deviation from True Value) of Spiked Sunflower Oil Samples

\begin{tabular}{|c|c|c|c|c|c|c|}
\hline \multirow[b]{2}{*}{$\begin{array}{l}\text { spiked } \mathrm{THC} / \mathrm{CBD} \text { ratio in } \\
\text { samples }\end{array}$} & \multirow[b]{2}{*}{$\begin{array}{l}\text { spiked absolute } \mathrm{THC} \text { in samples } \\
\left(\mu \mathrm{g} \mathrm{mL}^{-1}\right)\end{array}$} & \multirow[b]{2}{*}{$\begin{array}{l}\text { spiked absolute } \mathrm{CBD} \text { in samples } \\
\left(\mu \mathrm{g} \mathrm{mL}^{-1}\right)\end{array}$} & \multicolumn{2}{|c|}{ UHPLC-UV result } & \multicolumn{2}{|c|}{ AgPS-MS result } \\
\hline & & & $\begin{array}{l}\text { precision (\%) } \\
(N=3)\end{array}$ & $\begin{array}{c}\text { accuracy } \\
(\%)\end{array}$ & $\begin{array}{l}\text { precision }(\%) \\
(N=3)\end{array}$ & $\begin{array}{c}\text { accuracy } \\
(\%)\end{array}$ \\
\hline low $(0.004)$ & 0.200 & 50.0 & 2.3 & 8.3 & 11 & 14 \\
\hline medium (0.07) & 3.50 & 50.0 & 2.8 & 4.3 & 7.2 & -0.1 \\
\hline high $(0.3)$ & 15.0 & 50.0 & 1.2 & 1.3 & 1.7 & -2.7 \\
\hline
\end{tabular}


The LOD and LOQ of the AgPS-MS method to determine THC were determined at 6 and $20 \mathrm{ng} \cdot \mathrm{mL}^{-1}$, respectively. As the intensities in PS-MS are not very reproducible and quantification almost always relies on the use of an internal standard, santonin was used as the internal standard for THC. The $[\text { santonin }+\mathrm{Ag}]^{+}$adduct $(\mathrm{m} / z 353$ and 355$)$ produces characteristic $\mathrm{MS}^{2}$ fragments at $m / z 309$ and 311, which can be differentiated from the characteristic $\mathrm{MS}^{2}$ fragment of the $[\mathrm{THC}+\mathrm{Ag}]^{+}$adduct at $\mathrm{m} / z 313$ and thus used as the internal standard.

Application to Commercial CBD Oil Samples. Ten commercial samples were analyzed with the AgPS-MS and UHPLC-UV methods as benchmarks for their THC/CBD ratios (Figure 4A). Sunflower oil was used for the dilution of
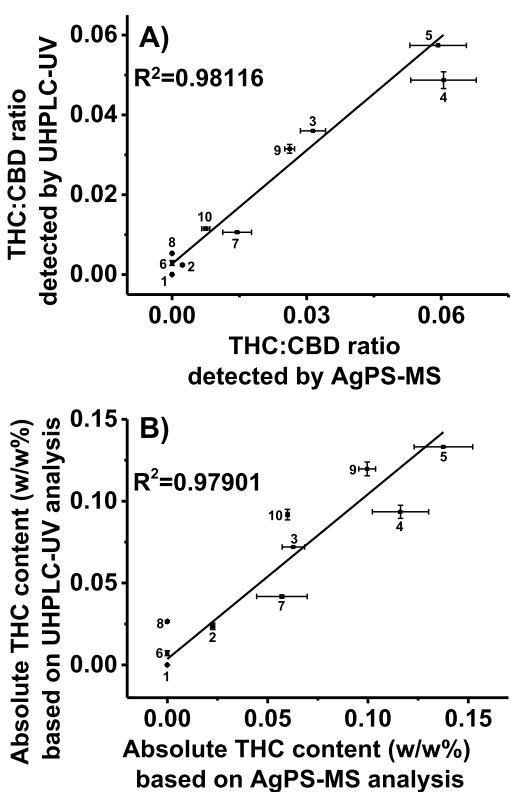

Figure 4. THC/CBD ratio detected by AgPS-MS vs UHPLC-UV methods (A); absolute THC content based on AgPS-MS vs UHPLCUV methods (B). Error bars represent standard deviation $(n=3)$.

CBD samples to $0.20 \%$ CBD. Because of the similar triacylglycerol composition as hemp oil, any matrix effects will be similar. The additional advantages of using sunflower oil for dilution instead of organic solvents are its availability, lack of toxicity, low cost, and environmental friendliness. Moreover, by assuming that the absolute CBD concentration as provided by the supplier is correct and taking the dilution factors into consideration, the absolute THC concentrations in these CBD oil products can be calculated. Although these do not provide direct absolute quantitative data, these estimates can serve as a screening tool to identify suspect samples that require further investigation to determine their compliance with legal standards. The clear advantage of this screening method is that it requires no addition of internal standards to the sample because of the use of the ratio between THC and CBD.

According to the results, in $\mathrm{CBD}$ oil 2, based on the detected ratio of $\mathrm{THC} / \mathrm{CBD}$ by $\mathrm{AgPS}-\overline{\mathrm{M}} \mathrm{S}$, the calculated absolute THC concentration is $0.0226 \pm 0.0010 \%$, which is almost identical with the UHPLC-UV result of $0.0236 \pm$ $0.0020 \%$. For CBD oil 6 and CBD oil 8, AgPS-MS is unable to detect the presence of THC because of their very low THC content, which was confirmed by the UHPLC-UV analysis result. For oils with an even lower THC content, like CBD oil 1, no THC signal was detected by either the UHPLC-UV or AgPS-MS method. Both methods revealed that CBD oils 3, $4,5,9$, and 10 contained a relatively high THC content and are actually over the Dutch legal limit of $0.05 \%$ if the declared CBD content is correct. In other words, if the actual CBD concentration is much lower than the declared value, the calculated THC concentration may be within the legal limit, but then the CBD content on the label is incorrect. In either case, it warrants further investigation by, for example, UHPLCUV analysis. For CBD oil_7, further analysis would be needed because the predicted result is around the legal limit, that is, $0.0571 \pm 0.0126 \%$ THC based on AgPS-MS and $0.0418 \pm$ $0.0008 \%$ based on UHPLC-UV detection.

In short, the developed method can quickly screen for the presence of THC in CBD oil by measuring the ratio of THC/ $\mathrm{CBD}$ and determine whether the THC content is below the legal limit based on the declared CBD content. Although from the perspective of linearity, RSD, and accuracy, the UHPLCUV methodology has a better performance, AgPS-MS greatly shortens the analysis time (from $17 \mathrm{~min}$ to less than $30 \mathrm{~s}$ ). Apart from this, AgPS-MS analysis does not require any mobile phase, as only $15 \mu \mathrm{L}$ of $\mathrm{MeOH}$ is needed for spraying, making it more environmentally friendly and cheaper. Moreover, for relative quantification analysis, that is, the determination of the THC/CBD ratio, the developed method does not require the use of deuterated standards, which are difficult to obtain and are expensive.

\section{CONCLUSIONS}

$\mathrm{Ag}(\mathrm{I})$-impregnated paper spray tandem MS allows for a fast distinction of THC and CBD, as well as for a reliable quantitative analysis of their concentration ratio. The method is based on a different complexation of THC and CBD with $\mathrm{Ag}(\mathrm{I})$ ions, leading in turn to different mass spectrometric fragmentation pathways. Samples with a wide range of THC/ $\mathrm{CBD}$ ratios $(\mathrm{THC} / \mathrm{CBD}=0.001-1)$ can be analyzed by the developed method within tens of seconds, requiring only minimal amounts of solvent. The good correspondence between the UHPLC-UV and AgPS-MS data of commercial CBD oils confirms the applicability of the method. Thus, it could be used for quality control of CBD oils. Another application area for this screening method could be the legal control of THC-poor hemp varieties for fiber production. Positive samples can then be retested in the lab by a validated quantitative method, such as UHPLC-UV.

\section{ASSOCIATED CONTENT}

\section{Supporting Information}

The Supporting Information is available free of charge at https://pubs.acs.org/doi/10.1021/acs.analchem.0c04270.

${ }^{1} \mathrm{H}$ NMR, TLC, and UHPLC analysis of THC and CBD standards; proposed CID fragmentation; argentation HPLC analysis; quantum chemical computations; influence of CID energy, temperature, and concentration on the product ion ratio; chromatographic and mass spectrometric analysis of THCA and CBDA; sample preparation and calibration curve of THC/CBD ratio constructed by AgPS-MS and UHPLC-UV; declared composition of commercial CBD oils; and AgPS-MS and UHPLC-UV analysis results of commercial CBD oils (PDF) 


\section{AUTHOR INFORMATION}

\section{Corresponding Authors}

Han Zuilhof - Key Laboratory of Phytochemical R\&D of Hunan Province and Key Laboratory of Chemical Biology \& Traditional Chinese Medicine Research of Ministry of Education, Hunan Normal University, Changsha 410081, China; Laboratory of Organic Chemistry, Wageningen University, Wageningen 6708 WE, The Netherlands; Department of Chemical and Materials Engineering, Faculty of Engineering, King Abdulaziz University, Jeddah 21589, Saudi Arabia; 10 orcid.org/0000-0001-5773-8506; Email: Han.Zuilhof@wur.nl

Gert IJ. Salentijn - Laboratory of Organic Chemistry, Wageningen University, Wageningen 6708 WE, The Netherlands; Wageningen Food Safety Research (WFSR), Wageningen University o Research, Wageningen 6700 AE, The Netherlands; 이이이.org/0000-0002-2870-9084; Email: Gert.Salentijn@wur.nl

\section{Authors}

Si Huang - Key Laboratory of Phytochemical R\&D of Hunan Province and Key Laboratory of Chemical Biology \& Traditional Chinese Medicine Research of Ministry of Education, Hunan Normal University, Changsha 410081, China; Laboratory of Organic Chemistry, Wageningen University, Wageningen 6708 WE, The Netherlands; (1) orcid.org/0000-0002-6792-086X

Frank W. Claassen - Laboratory of Organic Chemistry, Wageningen University, Wageningen 6708 WE, The Netherlands

Teris A. van Beek - Laboratory of Organic Chemistry, Wageningen University, Wageningen 6708 WE, The Netherlands

Bo Chen - Key Laboratory of Phytochemical R\&D of Hunan Province and Key Laboratory of Chemical Biology \& Traditional Chinese Medicine Research of Ministry of Education, Hunan Normal University, Changsha 410081, China; orcid.org/0000-0002-9926-4377

Jianguo Zeng - Hunan Key Laboratory of Traditional Chinese Veterinary Medicine, Hunan Agricultural University, Changsha 410128, China

Complete contact information is available at:

https://pubs.acs.org/10.1021/acs.analchem.0c04270

\section{Notes}

The authors declare no competing financial interest.

\section{ACKNOWLEDGMENTS}

The authors thank Prof. Michel Nielen (Wageningen Food Safety Research) for helpful discussions and acknowledge support from the National Natural Science Foundation of China (21775040, 21775041, and 21575040), the China Scholarship Council 2020 International Cooperation Training Program for Innovative Talents, the Aid Program for S\&T innovation research team in higher education institutions, the construction program of key disciplines of Hunan Province (2015JC1001), the project of Hunan Provincial Department of Education (17C0947), and the Hunan Province 100 experts project.

\section{REFERENCES}

(1) Chandra, S.; Radwan, M. M.; Majumdar, C. G.; Church, J. C.; Freeman, T. P.; ElSohly, M. A. Eur. Arch. Psychiatr. Clin. Neurosci. 2019, 269, 5-15.

(2) Comeau, Z. J.; Boileau, N. T.; Lee, T.; Melville, O. A.; Rice, N. A.; Troung, Y.; Harris, C. S.; Lessard, B. H.; Shuhendler, A. J. ACS Sens. 2019, 4, 2706-2715.

(3) Pertwee, R. G. Br. J. Pharmacol. 2008, 153, 199-215.

(4) Pertwee, R. G. Br. J. Pharmacol. 2009, 156, 397-411.

(5) Marinotti, O.; Sarill, M. J. Diet. Suppl. 2020, 17, 517.

(6) Hazekamp, A. Med. Cannabis Cannabinoids 2018, 1, 65-72.

(7) Manthey, J. Int. J. Drug Pol. 2019, 68, 93-96.

(8) Borges, G. R.; Birk, L.; Scheid, C.; Morés, L.; Carasek, E.; Kitamura, R. O. S.; Roveri, F. L.; Eller, S.; de Oliveira Merib, J.; de Oliveira, T. F. Forensic Toxicol. 2020, 38, 531-535.

(9) Zivovinovic, S.; Alder, R.; Allenspach, M. D.; Steuer, C. J. Anal. Sci. Technol. 2018, 9, 27.

(10) Hädener, M.; Gelmi, T. J.; Martin-Fabritius, M.; Weinmann, W.; Pfäfli, M. Int. J. Leg. Med. 2019, 133, 821-832.

(11) Amjadi, M.; Sodouri, T. J. Appl. Spectrosc. 2014, 81, 232-237.

(12) Kauppila, T. J.; Flink, A.; Laakkonen, U.-M.; Aalberg, L.; Ketola, R. A. Drug Test. Anal. 2013, 5, 186-190.

(13) Ifa, D. R.; Manicke, N. E.; Dill, A. L.; Cooks, R. G. Science 2008, 321,805 .

(14) Berman, P.; Futoran, K.; Lewitus, G. M.; Mukha, D.; Benami, M.; Shlomi, T.; Meiri, D. Sci. Rep. 2018, 8, 14280.

(15) Hädener, M.; Kamrath, M. Z.; Weinmann, W.; Groessl, M. Anal. Chem. 2018, 90, 8764-8768.

(16) Grossert, J. S.; Herrera, L. C.; Ramaley, L.; Melanson, J. E. J. Am. Soc. Mass Spectrom. 2014, 25, 1421-1440.

(17) Lévêque, N. L.; Héron, S.; Tchapla, A. J. Mass Spectrom. 2010, 45, 284-296.

(18) Acheampong, A.; Leveque, N.; Tchapla, A.; Heron, S. J. Chromatogr. A 2011, 1218, 5087-5100.

(19) Grade, H.; Winograd, N.; Cooks, R. G. J. Am. Chem. Soc. 1977, 99, 7725-7726.

(20) Cohen, L. H.; Gusev, A. I. Anal. Bioanal. Chem. 2002, 373, 571-586.

(21) van Beek, T. A.; Subrtova, D. Phytochem. Anal. 1995, 6, 1-19.

(22) Kaneti, J.; de Smet, L. C. P. M.; Boom, R.; Zuilhof, H.; Sudhölter, E. J. R. J. Phys. Chem. A 2002, 106, 11197-11204.

(23) Damyanova, B.; Momtchilova, S.; Bakalova, S.; Zuilhof, H.; Christie, W. W.; Kaneti, J. J. Mol. Struct.: THEOCHEM 2002, 589590, 239-249.

(24) Feider, C. L.; Krieger, A.; DeHoog, R. J.; Eberlin, L. S. Anal. Chem. 2019, 91, 4266-4290.

(25) Jackson, A. U.; Shum, T.; Sokol, E.; Dill, A.; Cooks, R. G. Anal. Bioanal. Chem. 2011, 399, 367-376.

(26) Wang, H.; Liu, J.; Cooks, R. G.; Ouyang, Z. Angew. Chem., Int. Ed. 2010, 49, 877-880.

(27) Liu, J.; Wang, H.; Manicke, N. E.; Lin, J.-M.; Cooks, R. G.; Ouyang, Z. Anal. Chem. 2010, 82, 2463-2471.

(28) Basuri, P.; Baidya, A.; Pradeep, T. Anal. Chem. 2019, 91, 71187124.

(29) Bambauer, T. P.; Maurer, H. H.; Weber, A. A.; Hannig, M.; Pütz, N.; Koch, M.; Manier, S. K.; Schneider, M.; Meyer, M. R. Talanta 2019, 204, 677-684.

(30) Damon, D. E.; Davis, K. M.; Moreira, C. R.; Capone, P.; Cruttenden, R.; Badu-Tawiah, A. K. Anal. Chem. 2016, 88, 18781884.

(31) Duvivier, W. F.; van Beek, T. A.; Pennings, E. J. M.; Nielen, M. W. F. Rapid Commun. Mass Spectrom. 2014, 28, 682-690.

(32) Gottardo, R.; Sorio, D.; Ballotari, M.; Tagliaro, F. J. Chromatogr. A 2019, 1591, 147-154.

(33) Cody, R. B.; Laramée, J. A.; Durst, H. D. Anal. Chem. 2005, 77, 2297-2302.

(34) Duvivier, W. F.; van Putten, M. R.; van Beek, T. A.; Nielen, M. W. F. Anal. Chem. 2016, 88, 2489-2496. 
(35) Shoeib, T.; Zhao, J.; Aribi, H. E.; Hopkinson, A. C.; Michael Siu, K. W. J. Am. Soc. Mass Spectrom. 2013, 24, 38-48.

(36) Sigsworth, S. W.; Castleman, A. W. J. Am. Chem. Soc. 1989, 111, 3566-3569.

(37) Jung, J.; Kempf, J.; Mahler, H.; Weinmann, W. J. Mass Spectrom. 2007, 42, 354-360.

(38) Eichler, M.; Spinedi, L.; Unfer-Grauwiler, S.; Bodmer, M.; Surber, C.; Luedi, M.; Drewe, J. Planta Med. 2012, 78, 686-691.

(39) Ariffin, A.; Bakar, J.; Tan, C.; Rahman, R.; Karim, R.; Loi, C. Food Chem. 2009, 114, 561-564.

(40) Scientific Working Group for Forensic Toxicology. J. Anal. Toxicol. 2013, 37, 452-474. 